\title{
INCORPORATING SAFETY INTO TARGETED PAVEMENT FRICTION DATA COLLECTION AND MAINTENANCE PROCEDURES
}

\author{
Ghazan Khan ${ }^{1}$, Andrea R. Bill², Kevan Shafizadeh ${ }^{3}$, David A. Noyce ${ }^{4}$ \\ ${ }^{1,3}$ Dept of Civil Engineering, California State University Sacramento, United States \\ ${ }^{2,4}$ Traffic Operations and Safety Laboratory, Dept of Civil and Environmental Engineering, \\ University of Wisconsin-Madison, United States
}

Submitted 1 May 2015; resubmitted 22 July 2015; accepted 25 October 2015

\begin{abstract}
The objective of this research was to develop a methodology for targeted pavement friction data collection based on the analysis of weather-related crashes. Furthermore, the aim was to identify threshold values of pavement friction characteristics indicating a significant impact on safety prompting the need for maintenance and improvements. Spatial analysis using Local Moran's $I$ statistic identified hotspots where pavement friction data were collected. A master database was assembled including Wisconsin State Trunk Network (STN) road attributes, hotspots of weather-related crashes, and pavement friction data collected based on hotspot analysis. The analysis results provide evidence in support of hotspot analysis as a viable procedure for targeted pavement friction data collection to enable efficiency and cost reductions. Classification tree analysis using GUIDE (Generalized, Unbiased, Interaction Detection and Estimation) algorithm was used to further explore the relationship between pavement friction characteristics and safety. Statistically significant hotspots were observed below a pavement friction number of approximately 57 and very high hotspots below a pavement friction number of approximately 42. The results indicate that pavement friction thresholds identified in the literature between 20 and 32 may be too low and that safety may be impacted at friction numbers as high as in the forties. The results also show differences in friction and safety for various types of pavement surfaces. The use of weather-related crashes provides a data-driven and cost-effective method of prioritizing locations for pavement friction data collection and maintenance. Results from this research can be readily used in initial steps of systemic road safety management procedures by practitioners.
\end{abstract}

Keywords: pavement friction; friction number; spatial analysis; spatial statistics; weather-related crashes; safety; classification tree; GUIDE.

\section{Background and Introduction}

The US has experienced approximately 30000 fatalities in road crashes for most years in the past five years (DoT $2012 b)$. Reducing fatal road crashes is one of the biggest challenges faced by transportation engineers in the US. There is a continuing need for researchers to develop cost effective and efficient means to improve road safety. 'Toward Zero Deaths: A National Strategy on Highway Safety' by the Federal Highway Administration (FHWA) as part of the current surface transportation bill, 'MAP21 - Moving Ahead for Progress in the 21st Century', calls for data-driven efforts towards traffic safety issues which serves as the foundation for this research (DoT 2015b, 2012a).

Approximately one-third of the crashes in the US occur during adverse weather conditions (rain, snow, fog, ice, etc.) (DoT 2015a). A potential contributory factor in such crashes is reduced pavement friction due to aging, wear, inadequate drainage during rainstorms, inadequate winter maintenance, and aberrations in the road surface such as potholes. Such conditions can impair vehicle stopping and maneuvering capabilities thereby increasing the risk of being involved in a crash. Transportation agencies across the nation collect road friction data periodically in order to determine which locations require maintenance and other improvements to restore adequate pavement friction.

The Wisconsin Department of Transportation (WisDOT) has been formally testing pavement friction on the entire State Trunk Network (STN) system since 1975. Different statistical models were developed through several research projects to predict the pavement friction characteristics over its service life (Noyce et al. 2007). After approximately 20 years of pavement 
friction testing, WisDOT determined that as long as the aggregates used during road construction met specified design standards, sufficient pavement friction would be available for the life of the pavement. In order to reduce costs and promote efficiency, WisDOT has decided to scale back on the comprehensive program of collecting pavement friction data for the entire STN highway system and focus on targeted data collection on needs basis and for research projects.

\section{Objectives and Study Design}

One of the challenges of the targeted approach to pavement friction data collection is the question of which road segments should be targeted? Therefore, the main objective of this research was to develop a methodology to identify specific highway segments where pavement friction data collection activities should be focused. Although there are many examples of hotspot analysis in the literature that employ various classical statistical methods, the idea was to identify road segments with high frequency of weather-related crashes associated with reduction in pavement friction using spatial statistical methods because such methods are able to analyze specific locations with respect to nearby locations of similar weather to identify changing trends and hotspots (De Luca et al. 2012; De Luca, Dell'Acqua 2014).

Another objective of this research was to examine the hotspots of weather-related crashes to identify threshold values of pavement friction and other characteristics that would indicate a significant impact on safety prompting the need for maintenance and improvements. The process of identifying and analyzing hotspots of weather-related crashes would help enhance the understanding of the relationship between pavement friction and safety while helping agencies reduce the financial burden of a comprehensive approach towards pavement friction data collection by using the a targeted approach.

Subsequent sections present details of the hotspot identification, which was completed in 2011 followed by targeted friction data collection by WisDOT in 2012 . Once the friction data had been processed, detailed analysis of hotspots for friction thresholds and additional characteristics was conducted. This paper highlights the analysis from these efforts.

\section{Literature Review}

Most of the latest literature concerning pavement friction data collection has been adequately summarized in the reports by Federal Highway Administration (FHWA) and Texas Department of Transportation (McGovern et al. 2011; Long et al. 2014). Details on the use of weather-related crashes and threshold values for pavement friction vary greatly amongst various states. California analyses the locations of wet weather crashes periodically as part of overall safety studies to identify specific locations for treatment. Pavement friction data is collected periodically for all roads in the state with no specific threshold values. Florida also collects pavement friction data on all roads on a three-year rotation. However, locations with friction number 28 (FN40R) or less for posted speed limit of 45 miles per hour or less, and 30 (FN40R) or less for posted speed greater than 45 miles per hour are specially scrutinized. Michigan and New York use a friction number threshold of 30 and 32 or less, respectively, for prioritizing locations. New York also prioritizes locations if there are at least six wet road crashes during a two-year period in rural areas and at least 10 in urban areas with at least $35 \%$ of the total crashes occurring on wet road conditions. Virginia uses the criteria of a minimum three wet weather crashes, each separated by less than 0.2 miles and proportion of wet weather crashes at least $20 \%$ higher than the ratio for all roads in the area. Locations with a friction number less than 20 (SN40S) are flagged for review by the districts. However, a more recent study conducted by National Highway Traffic Safety Administration's (NHTSA's) Vehicle Research and Testing Center (VTRC) and Virginia Tech has recommended the use of a higher value (25-30) (McGovern et al. 2011; Long et al. 2014).

Researchers and agencies also use regression and other statistical models to predict the deterioration in pavement characteristics over time (Dell'Acqua et al. 2011; De Luca, Dell'Acqua 2014). WisDOT employs empirical models (Russell Model), which were developed to predict the friction number as a function of asphalt material properties, service age of pavement, traffic mix, and volume, as well as climate variations (Noyce et al. 2007; Vadakpat 1994):

for Hot Mix Asphalt (HMA):

$$
\begin{aligned}
& F N=414.4-0.00075 \cdot D^{2}- \\
& 1.45 \cdot \ln (L A V P)+0.245 \cdot L A W E A R ;
\end{aligned}
$$

for Portland Cement Concrete (PCC):

$$
\begin{aligned}
& \ln (F N)=3.99-0.0419 \cdot \ln (L A V P)- \\
& 0.00129 \cdot D+0.00474 \cdot H V,
\end{aligned}
$$

where: $F N$ - friction number calculated at $40 \mathrm{mph}$; $D$ percent dolomite in the asphalt mix; LAVP - lane accumulated vehicle passes; $L A W E A R$ - Los Angeles abrasion test result (ASTM C535-12); $H V$ - percent heavy vehicles in the design lane.

Based on empirical knowledge and past experience, WisDOT uses a desirable minimum friction number of 35 as a threshold for pavement monitoring and improvements (Noyce et al. 2007).

From the literature, it is evident that most states base their analyses on weather-related crashes and some level of threshold values in prioritizing maintenance and reconstruction operations, but there is no consistent practice associating pavement friction and safety. Therefore, there is a need to further explore the subject matter in this research. 


\section{Identification of Weather Crash Hotspots}

\subsection{Data Collection and Processing}

The first step of this research study was to develop a methodology for the identification of hotspots of weather-related crashes where pavement friction data collection could be focused. Three years of Wisconsin crash data on STN roads (2008 through 2010) were used in consultation with WisDOT officials. A shapefile of crash locations was generated by WisDOT using intersection or milepost location, distance of the crash from intersection/milepost (in increments of one hundredth of a mile), and STN specific reference point tables identifying specific locations on the system, thus allowing researchers with an accurate position of the crashes. Animal and intersection-related crashes were removed, resulting in a total of 72792 crashes for the three year period. Based on information in the crash report forms, weather-related crashes were identified considering the presence of rain or snow at the time of crash or a checkmark indicating adverse weather as a contributing factor resulting in 31571 weather-related crashes for the three year period between 2008 and 2010 .

The total and weather-related crashes were aggregated on individual segments of the Wisconsin MetaManager (MM) road database (separately for each direction of divided road sections) which contains more than 19000 homogenous segments of the Wisconsin STN roads along with detailed attributes related to safety, mobility, and infrastructure. A weather-related crash rate was calculated to normalize crashes by exposure to facilitate the comparisons between different MM segments. The weather-related crash rate was defined as follows:

$$
x_{i}=\frac{\sum W C_{i}}{\sum T C_{i}},
$$

where: $x_{i}$ - weather-related crash rate for MM segment $i$; $W C_{i}$ - sum of weather-related crashes on MM segment $i$; $T C_{i}$ - sum of all crashes on MM segment $i$.

The reason for using the sum of all crashes as exposure instead of traffic volume was significant variability in Annual Daily Traffic (ADT) data over the three-year analysis period and the non-linear relationship between weather-related crashes and ADT in Wisconsin from past research (Qin et al. 2007).

\subsection{Methodology}

Although patterns in spatial data can be visually analyzed through mapping frequency, mean, or proportions, spatial statistical methods go one-step further in utilizing advanced statistical methods to quantify and discern patterns in spatial data. The ability to visually discern patterns can be limiting, therefore spatial statistical methods can provide a quantifiable measure of spatial patterns rather than a pre-defined ranking or number.

There are several spatial statistical methods available for analyzing patterns to identify hotspots. Some methods can identify hotspots as clusters of high or low attribute values (Getis-Ord $\mathrm{Gi}^{*}$ statistic) while others identify clusters of similar or dissimilar values (Anselin's Local Moran I statistic) (Getis, Ord 1992; Anselin 1995; Ord, Getis 1995). In this research, the Local Moran's $I$ statistic was used because it has the added advantage of classifying spatial patterns by high or low attribute values besides similar and dissimilar values as defined below:

- HH - high weather-related crash rate location surrounded by other locations with high weather-related crash rate;

- HL - high weather-related crash rate location surrounded by other locations with low weatherrelated crash rate;

- LH - low weather-related crash rate location surrounded by other locations with high weatherrelated crash rate;

- LL - low weather-related crash rate location surrounded by other locations with low weatherrelated crash rate.

The Local Moran's I statistic calculated an index value $I$ and associated $z$-score for each feature (MM segment) analyzed. A positive index value $I$ for a feature indicates that it is clustered with features of similar attribute values, and a negative value indicates that the feature is clustered by dissimilar values. Z-scores are measures of statistical significance indicating whether the similarity or dissimilarity in attribute values between the feature and its neighbors is greater than one would expect simply by chance (Getis, Ord 1992). The more positive or negative the $z$-score, the more significant the results are. The Local Moran's I statistic can be presented as follows:

$$
I_{i}=\frac{x_{i}-\bar{x}}{S^{2}} \sum_{j=1}^{N} w_{i j}\left(x_{j}-\bar{x}\right),
$$

where:

$$
S^{2}=\frac{\sum_{j=1, j \neq i}^{N} x_{j}^{2}}{N-1}-\bar{x}^{2} ;
$$

$I_{i}$ - Local Moran's $I$ statistic; $x_{i}$ - weather-related crash rate of MM segment $i ; x_{j}$ - weather-related crash rate of neighboring MM segment $j ; w_{i j}$ - spatial weight matrix around $i$ for all sites $j ; N$ - number of weighted points, each representing weather-related crash rate for each MM segment.

In Eq. (4), $w_{i j}$ represents the nature and extent of the neighborhood around $x_{i}$, which defines the relationship between locations. The relationship is based on the nature of attributes (weather-related crashes) being analyzed. The spatial weight matrix $w_{i j}$ in this research was based on the inverse distance function, which indicates a decreasing influence of the spatial relationship with increasing distance because weather tends to be similar at closer locations than at locations further away.

The inverse distance function represents the nature of the relationship between neighboring locations, but does not define an actual extent of the neighborhood, which is more challenging. The Local Moran's $I$ and Getis-Ord $\mathrm{Gi}^{*}$ statistics are local spatial statistical methods which can identify local spatial patterns in a 
predefined neighborhood. Conversely, global spatial statistical methods, such as Global Moran's I statistic, can measure the overall intensity of clustering (hotspots) in the study area (Getis, Ord 1992). The idea in this research was to calculate the Global Moran's I statistic at various distance scales, identifying the distance where spatial patterns (clustering) peak. The intensity of the patterns (clustering) is represented by the $z$-score. The peak distance was used to define the neighborhood for the Local Moran's I statistic. The analysis was conducted using ESRI ArcGIS ${ }^{\mathrm{TM}}$ software.

\subsection{Results and Discussion}

\subsubsection{Global Moran's I Statistic}

The result of Global Moran's I statistic is presented in Fig. 1 where the $x$-axis shows the distance scales or bandwidths at which the statistic was calculated. A total of 10 distance bandwidths were analyzed in increments of $1 \mathrm{~km}$ starting at $5 \mathrm{~km}$. The starting bandwidth of $5 \mathrm{~km}$ was selected because the minimum distance required to ensure that all MM segments have at least 1 neighbor was $4.3 \mathrm{~km}$. The $y$-axis represents the $z$-score indicating the intensity of clustering (hotspots) of weather-related crash rate in the study area. Fig. 1 shows that clustering peaks at a distance of $12 \mathrm{~km}$, which was selected as the size of the neighborhood along with inverse distance function to define the spatial weight matrix for Local Moran's I statistic.

\subsubsection{Local Moran's I Statistic}

The result of Local Moran's I statistic identified different types of statistically significant (90\% confidence level, $z$-score \pm 1.645$)$ spatial patterns of weather-related crashes in Wisconsin, which were defined previously. In this research, the primary interest was in $\mathrm{HH}$ and HL locations (MM segments) because HH MM segment represents locations of high weather-related crash rate surrounded by other high weather-related crash rate MM segments, whereas HL MM segment represents locations of high weather-related crash rate surrounded by low weather-related crash rate MM segments. Both $\mathrm{HH}$ and HL MM segments thus represent hotspots of weather-related crashes signifying systematic weatherprone locations with respect to road safety. The identification of HH and HL MM segments provides an objective data-driven method of prioritizing locations for targeted pavement friction data collection efforts.

Based on the spatial weight matrix of inverse distance function, $12 \mathrm{~km}$ cut-off distance, and $90 \%$ confidence level, the result of Local Moran's I statistic identified more than 2000 hotspots (HH and HL MM segments) throughout the state of Wisconsin. In consultations with WisDOT officials, MM segments with three or less crashes were removed, resulting in a total of 304 $\mathrm{HH}$ and $188 \mathrm{HL}$ hotspots. Fig. 2 illustrates the result of Local Moran's I statistic showing statistically significant spatial patterns (hotspots) in weather-related crashes (using weather-related crash rate). MM segments which were not part of a statistically significant hotspot $(\mathrm{HH}$ and HL) are not shown in Fig. 2.
The result presented in Fig. 2 was shared with WisDOT officials for further consultations. In order to prepare a realistic plan for targeted pavement friction data collection, contiguous MM segments on various STN roads were identified taking in to account the hotspots as shown in Fig. 2, local knowledge and experience, and potential future reconstruction projects. Although the main interest was in $\mathrm{HH}$ and HL hotspots, the contiguous segments included LL and LH locations which were also of interest providing a basis for comparing and contrasting the discerning characteristics that may indicate factors affecting weather-related crashes. In the end, a total of $3388 \mathrm{MM}$ segments based around the hotspots in Fig. 2 on various STN roads in Wisconsin were identi-

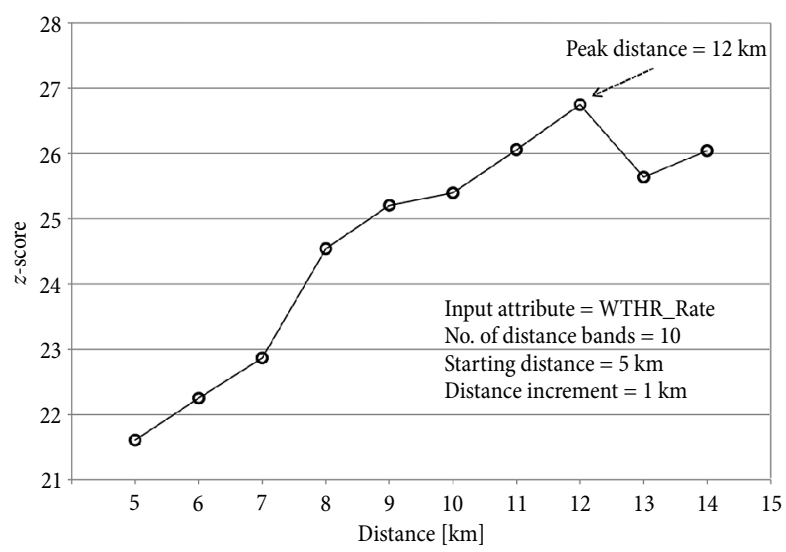

Fig. 1. Result of Global Moran's I statistic showing distance bandwidth of peak clustering of weather-related crash rate in Wisconsin

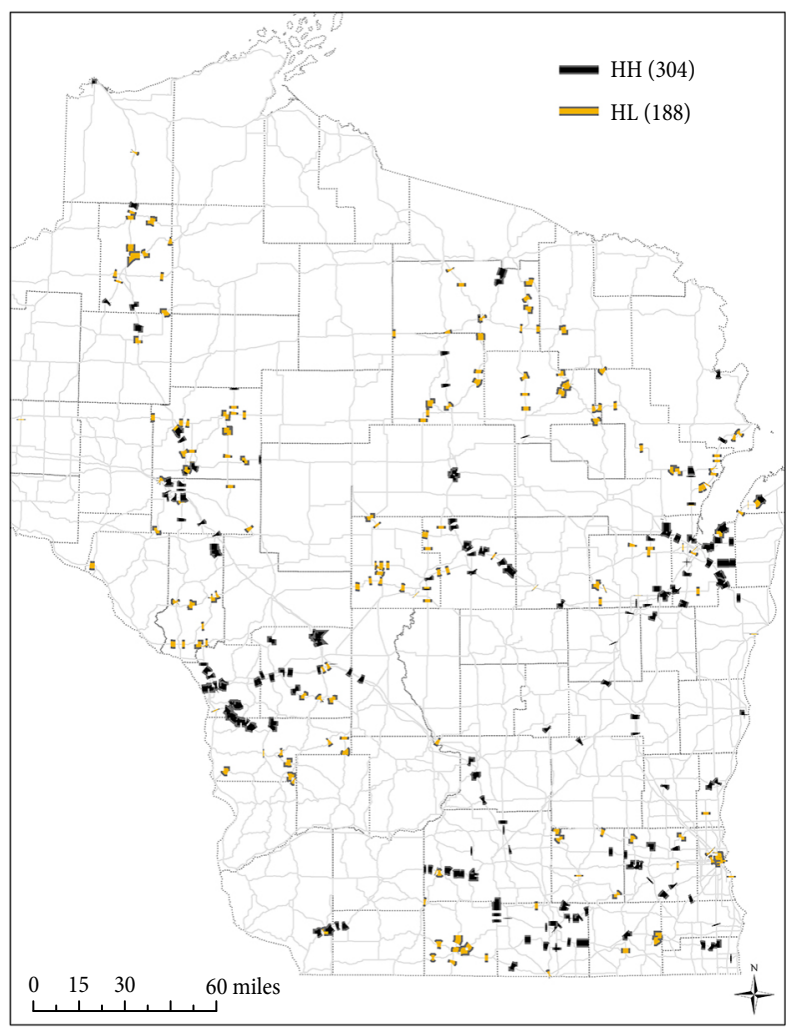

Fig. 2. Local Moran's I hotspots of weather-related crashes in Wisconsin (greater than three crashes per MM segment) 
fied for targeted pavement friction data collection. The segments included $304 \mathrm{HH}, 188 \mathrm{HL}, 93 \mathrm{LH}$, and $120 \mathrm{LL}$ statistically significant spatial patterns and 2683 nonsignificant MM segments, which are illustrated in Fig. 3.

\section{Analysis of Friction Data and Hotspot Characteristics}

In June 2012, WisDOT contracted Dynatest Consulting Inc. to conduct targeted friction testing on the 3388 MM segments (representing 3243 miles) in 46 counties of Wisconsin based on the results of Local Moran's I hotspot analysis. All testing was performed in accordance with the procedures detailed in ASTM E274 and using the ASTM E501 standard rib tire (Gokhale, Bischoff 2012). Horizontal curves, intersections, and ramps were not tested as per WisDOT directives (Gokhale, Bischoff 2012). Details of the data collection process are presented in the final report submitted to WisDOT by the contractor (Gokhale, Bischoff 2012).

\subsection{Data Collection and Processing}

A number of data were collected as part of the targeted pavement friction data collection process including pavement friction number at 40 miles per hour (SN40R), Mean Profile Depth (MPD), and Estimated Texture Depth (ETD). Friction data collected was integrated with relevant variables of the Wisconsin MM database to create a master database used in the analysis of hotspots of weather-related crashes in Wisconsin as presented in Table.

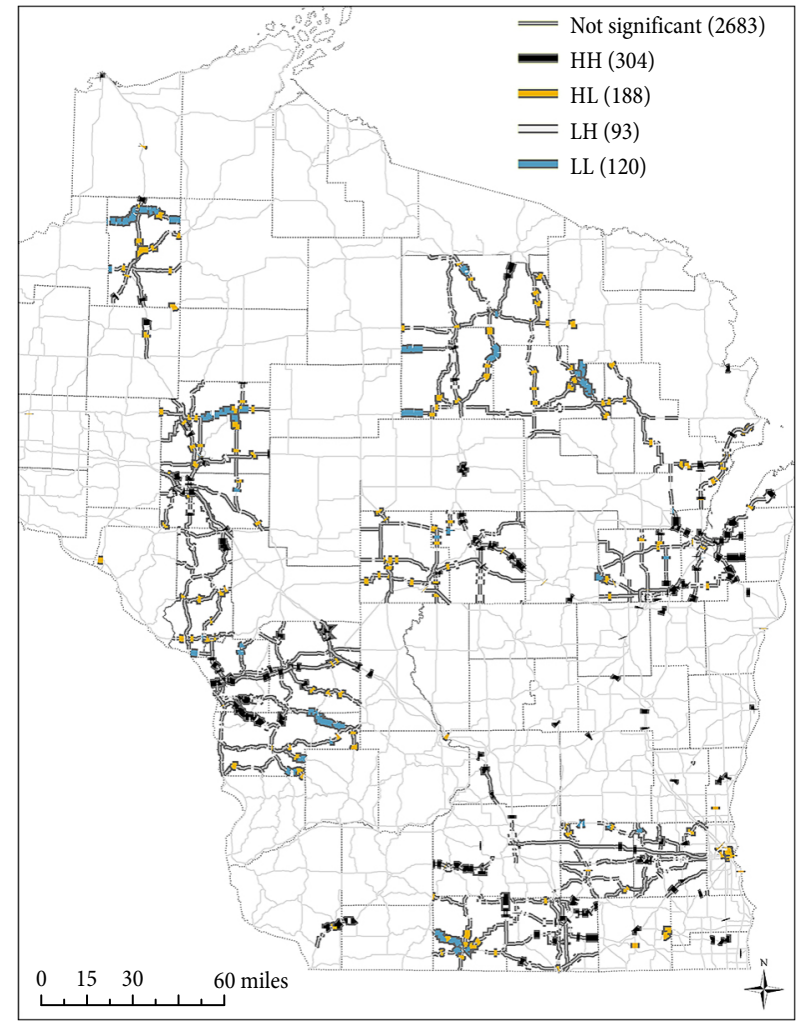

Fig. 3. Contiguous MM segments of Wisconsin STN roads for targeted pavement friction data collection (including Local Moran's I hotspots of weather-related crashes)

Table. Variables used in analysis of hotspots of weather-related crashes in Wisconsin

\begin{tabular}{|c|c|c|c|c|}
\hline Variable name & Variable description & Variable categories & Description & MM segment count \\
\hline \multirow{5}{*}{ COType 1} & \multirow{5}{*}{ Types of Local Moran's I spatial patterns } & $\mathrm{HH}$ & High/High & 304 \\
\hline & & $\mathrm{HL}$ & High/Low & 188 \\
\hline & & LH & Low/High & 93 \\
\hline & & LL & Low/Low & 120 \\
\hline & & NS & Not significant & 2683 \\
\hline \multirow{5}{*}{ Region } & \multirow{5}{*}{ WisDOT region } & NC & North Central & 729 \\
\hline & & $\mathrm{NE}$ & North East & 529 \\
\hline & & NW & North West & 725 \\
\hline & & SE & South East & 263 \\
\hline & & SW & South West & 1142 \\
\hline \multirow{2}{*}{ FLCASSb } & \multirow{2}{*}{ Area type } & Urban & Urban & 928 \\
\hline & & Rural & Rural & 2460 \\
\hline \multirow{3}{*}{ BroadSurf } & \multirow{3}{*}{ Pavement surface type } & $\mathrm{AC}$ & Hot mix asphalt & 2413 \\
\hline & & PCC & Concrete & 891 \\
\hline & & SMA & Stone matrix asphalt & 84 \\
\hline Variable name & Variable description & Mean & Median & Std. dev. \\
\hline WTHR_Rate & Weather crash rate & 0.40 & 0.36 & 0.37 \\
\hline Sum_TOT & Total number of crashes per MM segment & 3.93 & 2.00 & 6.53 \\
\hline Sum_WTHR & $\begin{array}{l}\text { Total number of weather-related crashes } \\
\text { per MM segment }\end{array}$ & 1.97 & 1.00 & 3.29 \\
\hline TRKYR_1 & Percentage of trucks & 11.43 & 10.00 & 5.59 \\
\hline Age & Pavement age [years] & 12.87 & 12.00 & 8.69 \\
\hline SN40R & Pavement friction humber & 50.63 & 51.80 & 7.45 \\
\hline Avg_MPD & Average MPD for MM segment & 0.02 & 0.02 & 0.02 \\
\hline Avg_ETD & Average ETD for MM segment & 0.02 & 0.03 & 0.01 \\
\hline
\end{tabular}


The master database was used in further analysis to explore the relationship between pavement friction characteristics and safety to identify threshold values that would indicate significant changes in safety. Exploratory analysis of the master database revealed interesting trends, which are presented in Fig. 4. Fig. 4a shows a plot between MPD and ETD variables as almost a straight line, which was expected given that both variables represent similar characteristics of the pavement surface texture. In this research, it was decided to use MPD as the variable representing pavement surface texture. Fig. $4 \mathrm{~b}$ shows a nearly linear relationship between weather-related and total crashes on MM segments, which justifies the use of total crashes as the measure of exposure. Fig. 4c illustrates an increasing trend in pavement friction number as the MPD of pavement increases, which is an important consideration in identifying threshold values impacting safety.

Fig. 5 displays the relationship between different types of hotspots of weather-related crashes, pavement friction number, and MPD using boxplots and mean values. Fig. $5 \mathrm{a}$ shows that the pavement friction number is the lowest for hotspots of weather-related crashes (HH and HL MM segments) except for locations which do not show statistically significant spatial patterns. Similarly, Fig. 5b also illustrates a similar trend for MPD, which is lowest for hotspots of weather-related crashes. The results in Fig. 5 indicate that there is a direct relationship between declining safety and pavement friction, validating the use of hotspot identification as a viable methodology for targeted pavement friction data collection. Furthermore, the direct relationship means that a

a)

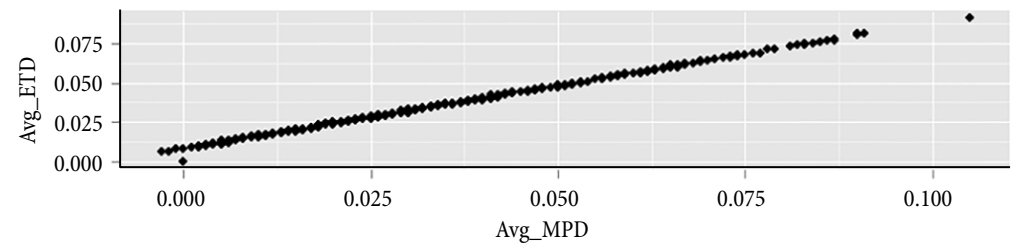

b)

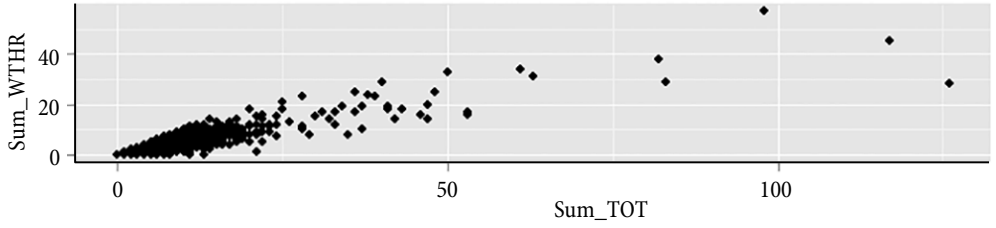

c)

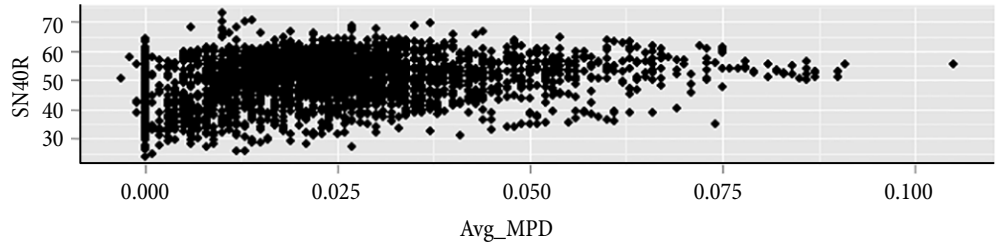

Fig. 4. Scatterplots of crashes and pavement friction characteristics
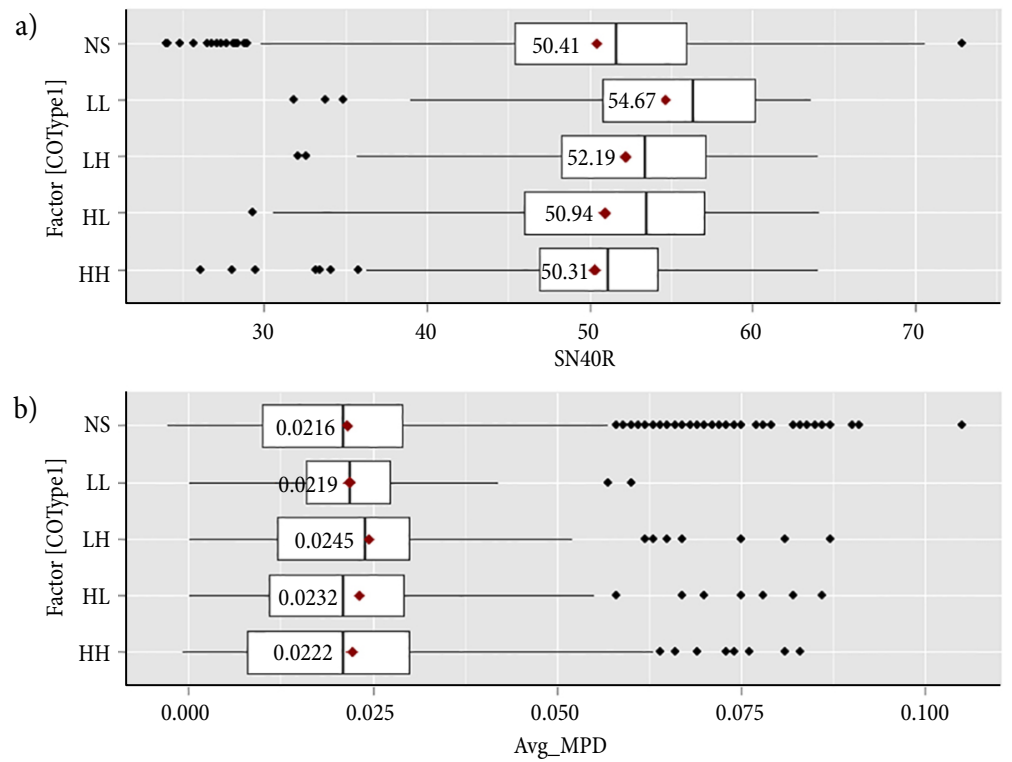

Fig. 5. Boxplots of pavement friction characteristics and types of hotspots of weather-related crashes 
threshold value can be determined beyond, which safety deteriorates significantly. Such locations should be prioritized for maintenance and other improvements.

In view of information presented in Figs 4 and 5, further analysis between safety (hotspots of weather-related crashes) and pavement friction was conducted to identify threshold values and discerning characteristics of MM segments with pavement friction issues. Classical regression methods were not well suited for analysis due to non-compliance of assumptions in the data and categorical dependent variable (hotspots of weatherrelated crashes). The best choice in this case was to use non-parametric methods, specifically classification trees, which do not require the stringent assumptions associated with classical regression methods.

\subsection{Methodology}

\subsubsection{Classification Tree Using GUIDE}

Classification trees are machine-learning methods for constructing prediction models through recursive partitioning of data, which can be graphically represented as a decision tree. In each of the recursive iteration, the aim is to find a factor and its partitioning value that results in the most homogeneous subset. There are several algorithms in literature, which implement classification trees with different strengths and weaknesses (Loh 2011). The classification tree algorithm used in this research was GUIDE (Generalized, Unbiased, Interaction Detection and Estimation). GUIDE offers advantages in terms of unbiased splits (removing bias in splits due to large differences in sample sizes) and robust method of tree pruning, as compared with other regression tree algorithms e.g. Classification And Regression Tree (CART) (Loh 2011, 2002). GUIDE also offers several options to select the appropriate tree size based on cross validation techniques.

For GUIDE analysis, a unit misclassification cost matrix was used as a means of assigning equal weight to different categories of the dependent variable during classification. The interpretation of a classification tree model becomes increasingly difficult with large number of splits and terminal nodes. In addition, over-fitting can occur when the number of split levels approaches the sample size in the absence of constraints, e.g. one of the trees in this research without pruning resulted in more than 50 terminal nodes. GUIDE minimizes this issue by allowing the user to fit complex models at nodes reducing the need for additional splits and nodes. Additionally, GUIDE uses user specified V-fold cross-validation for pruning to develop optimal tree structures. In this analysis, the optimal tree structure was selected by GUIDE using ten-fold cross-validation and the mean of cross-validation estimates.

\subsection{Results and Discussion}

The goal of classification tree analysis was to develop simple models identifying breakpoints or threshold values in data indicating significant changes in safety with respect to changes in pavement friction and related characteristics. The GUIDE Classification Tree for hotspot analysis of weather-related crashes in Wisconsin using unit misclassification cost matrix is presented in Fig. 6, where '?' represents missing or unknown data. The dependent variable was the different types of hotspots $(\mathrm{HH}, \mathrm{HL}, \mathrm{LH}$, and $\mathrm{LL}$ ); and independent variables are presented in Table.

Fig. 6 shows both graphical and textual representation of the GUIDE Classification Tree structure. Splitting variables are shown besides each intermediate or root node where an observation (individual MM segment) goes to the left only if the condition for the variable besides a specific node is met. For example, if the value of pavement friction number (SN40R) is less than 57.85 at the root node, all cases are transferred to node 2 and the rest are transferred to node 3 . The predicted class is shown below each terminal node representing the dominant trait (type of hotspot) of the homogenous subset of data. The numbers beside each terminal node represent the class sample size and misclassified observations.

The GUIDE Classification Tree result in Fig. 6 shows that for pavement friction number greater than 57.85, there are no hotspots in weather-related crashes except when pavement type is PCC. Conversely, for a pavement friction number of less than $57.85, \mathrm{HH}$ hotspots are observed for the following conditions:

- MM segments of PCC and SMA pavement types;

- AC pavement type in urban area;

- AC pavement type in rural area with MPD less than 0.012;

- AC pavement type in rural area with MPD greater than 0.012 and pavement friction number less than 53.75 .

However, MM segments of AC pavement type in rural area where MPD is greater than 0.012 and pavement friction number is between 53.75 and 57.85 shows HL hotspots. The results in Fig. 6 indicate a pavement friction threshold value of 57.85 beyond, which there are significant hotspots in weather-related crashes, except in the case of PCC pavement type, which requires further investigation.

The result presented in Fig. 6 shows a classification tree with five levels and seven terminal nodes. As the tree grows bigger, the model becomes complex, difficult to interpret and use in the real world. Therefore, efforts were made to simplify the model by removing the functional classification (FCLASSb) variable through trial and error. The result of GUIDE Classification Tree using weather-related crash rate in Wisconsin and no functional classification variable is presented in Fig. 7. Fig. 7 shows that for SMA and PCC pavement types, friction values less than 56.06 are critical resulting in $\mathrm{HH}$ hotspots. However, for $\mathrm{AC}$ pavement, friction value of less than 41.75 results in HL hotspot. HL hotspots are potentially more critical since it represents a MM segment with weather-related crash rate significantly higher than adjacent locations as compared with $\mathrm{HH}$ hotspot 
which represents a high weather-related crash rate but comparable with adjacent locations. The result in Fig. 7 shows two threshold values of pavement friction for different types of pavement surfaces beyond, which safety is significantly deteriorated. The numbers can be used to analyze the need for targeted pavement maintenance and improvement activities in the future. It should be noted that for pavement friction numbers above 56.05, there are no hotspots except in the case of PCC pavement type, which requires further investigation.

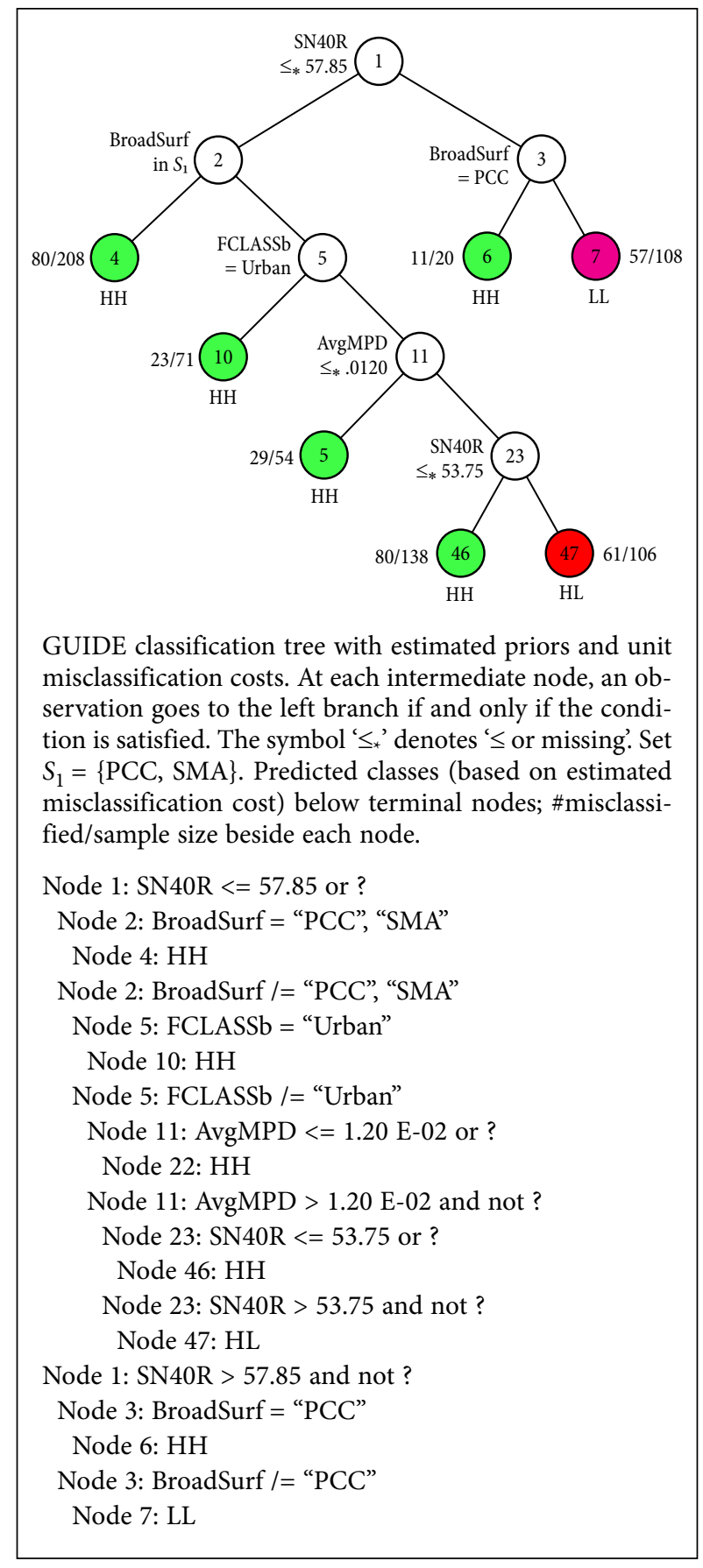

Fig. 6. GUIDE Classification Tree result of hotspots of weather-related crash rate in Wisconsin

\section{Summary and Conclusions}

The objective of this research was to develop a methodology for targeted pavement friction data collection based on the analysis of weather-related crashes and to identify threshold values of pavement friction characteristics that would indicate a significant impact on safety prompting the need for maintenance and improvements. Results of spatial analysis of weather-related crashes using Local Moran's I method identified spatial patterns ( $\mathrm{HH}$ and HL hotspots of MM segments) providing an objective and data-driven method of prioritizing locations for targeted pavement friction data collection.

After completion of targeted pavement friction data collection, a master database was assembled including friction, hotspot, and other MM segment data attributes. Pavement friction numbers and MPD associated with hotspots showed a direct relationship in Fig. 5 which concludes that hotspot identification method using weather-related crashes is viable for targeted pavement friction data collection.

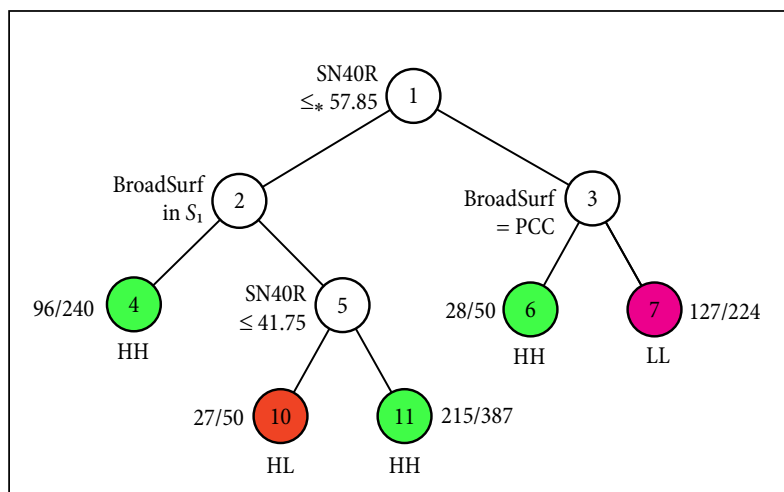

GUIDE classification tree with estimated priors and unit misclassification costs. At each intermediate node, an observation goes to the left branch if and only if the condition is satisfied. The symbol ' $\leq{ }^{\star}$ ' denotes ' $\leq$ or missing. Set $S_{1}=\{$ PCC, SMA $\}$. Predicted classes (based on estimated misclassification cost) below terminal nodes; \#misclassified/sample size beside each node.

Node 1: SN40R $<=56.05$ or ?

Node 2: BroadSurf = "PCC", "SMA"

Node 4: $\mathrm{HH}$

Node 2: BroadSurf /= "PCC" "SMA"

Node 5: $\mathrm{SN} 40 \mathrm{R}<=41.75$

Node 10: HL

Node 5: SN40R $>41.75$ or ?

Node 11: $\mathrm{HH}$

Node 1: SN40R > 56.05 and not?

Node 3: BroadSurf = "PCC"

Node 6: $\mathrm{HH}$

Node 3: BroadSurf /= "PCC"

Node 7: LL

Fig. 7. GUIDE Classification Tree result of hotspots of weather-related crash rate in Wisconsin without functional class variable 
The master database was used in further analysis to explore the relationship between pavement friction characteristics and hotspots to identify threshold values that would indicate significant changes in safety. The results of GUIDE Classification Trees in Figs 6 and 7 show statistically significant hotspots below a pavement friction number of approximately 57 and very high hotspots below pavement friction number of approximately 42 . These results conclude that the general pavement friction thresholds as identified in the literature, which ranged from 20 to 32 may be too low and that there is a possibility of safety being impacted even at friction numbers as high as in the forties. Furthermore, the results also show the differences in friction and safety for various types of pavement surfaces. The results of GUIDE Classification Trees illustrate a simple model, which is easy to interpret and provides thresholds regarding safety of weather-related crashes related to pavement friction. Such results can be readily used in initial steps of systemic road safety management procedures by practitioners.

The Local Moran's I and GUIDE Classification Tree methods and processes described in this research were based on off-the-shelf software and techniques which are easily implementable and repeatable in the future. Classification trees are easy to use and without restrictions in terms of underlying assumptions associated with classical regression methods. However, classification tree methods do require some trial and error to produce the best (simplest), most informative, and practical model.

The methods used in this research pave the way for targeted pavement data collection and maintenance using data driven approach promoting efficiency and cost effectiveness. One limitation of this research is the unavailability of rainfall intensity data, which could have safety implications for various types of pavement surfaces (Dell'Acqua et al. 2012). Nevertheless, the process of identifying and analyzing hotspots helped enhance the understanding of the relationship between pavement friction and weather-related crashes while helping agencies reduce the financial burden of a comprehensive approach by using a targeted approach. The methods and processes described in this research will also enable agencies to comply with MAP-21 (DoT 2012a) requirements in terms of data-driven approach towards safety improvements.

\section{Acknowledgements}

This research was funded by the Provost's Research Incentive Fund program at California State University Sacramento. The authors would like to recognize the support of Rebecca Szymkowski and Brian Porter from BTO, Scott Janowiak from BSHP, and Deb Bischoff from BTS at Wisconsin Department of Transportation (WisDOT) for providing the detailed friction data and associated final report.

\section{References}

Anselin, L. 1995. Local indicators of spatial association - LISA, Geographical Analysis 27(2): 93-115.

http://doi.org/10.1111/j.1538-4632.1995.tb00338.x

ASTM C535-12. Standard Test Method for Resistance to Degradation of Large-Size Coarse Aggregate by Abrasion and Impact in the Los Angeles Machine.

ASTM E274. Standard Test Method for Skid Resistance of Paved Surfaces Using a Full-Scale Tire.

ASTM E501. Standard Specification for Standard Tire For Pavement Skid-Resistance Tests.

De Luca, M.; Dell'Acqua, G.; Lamberti, R. 2012. Road safety analysis using operating speeds: case studies in Southern Italy, Procedia - Social and Behavioral Sciences 53: 702- 710. http://doi.org/10.1016/j.sbspro.2012.09.920

De Luca, M; Dell'Acqua, G. 2014. Runway surface friction characteristics assessment for Lamezia Terme airfield pavement management system, Journal of Air Transport Management 34: 1-5. http://doi.org/10.1016/j.jairtraman.2013.06.015

Dell'Acqua, G.; De Luca, M.; Lamberti, R. 2011. Indirect skid resistance measurement for porous asphalt pavement management, Transportation Research Record: Journal of the Transportation Research Board 2205: 147-154. http://doi.org/10.3141/2205-19

Dell'Acqua, G.; De Luca, M.; Mauro, R.; Russo, F. 2012. Freeway crashes in wet weather: the comparative influence of porous and conventional asphalt surfacing, Procedia - Social and Behavioral Sciences 54: 618-627. http://doi.org/10.1016/j.sbspro.2012.09.779

DoT. 2015a. Road Weather Management: How Do Weather Events Impact Roads? Federal Highway Administration, US Department of Transportation (DoT). Available from Internet: http://www.ops.fhwa.dot.gov/weather/q1_roadimpact.htm

DoT. 2015b. Toward Zero Deaths: A National Strategy on Highway Safety. 2015. Federal Highway Administration, US Department of Transportation (DoT). Available from Internet: http://safety.fhwa.dot.gov/tzd

DoT. 2012a. MAP-21 - Moving Ahead for Progress in the 21st Century. Federal Highway Administration, US Department of Transportation (DoT). Available from Internet: http:// www.fhwa.dot.gov/map21/factsheets/hsip.cfm

DoT. 2012b. Traffic Safety Facts 2012: A Compilation of Motor Vehicle Crash Data from the Fatality Analysis Reporting System and the General Estimates System. DOT HS 812032. National Highway Traffic Safety Administration, US Department of Transportation (DoT). 248 p. Available from Internet: http://www-nrd.nhtsa.dot.gov/Pubs/812032.pdf

Getis, A.; Ord, J. K. 1992. The analysis of spatial association by use of distance statistics, Geographical Analysis 24(3): 189206. http://doi.org/10.1111/j.1538-4632.1992.tb00261.x

Gokhale, S. V.; Bischoff, D. 2012. Statewide Pavement Friction Testing 2012. Report No WI-06-12. Wisconsin Department of Transportation, Madison, WI. 46 p. Available from Internet: http://wisconsindot.gov/documents2/research/BTSWI-06-12-final-report.pdf

Loh, W.-Y. 2011. Classification and regression trees, Wiley Interdisciplinary Reviews: Data Mining and Knowledge Discovery 1(1): 14-23. http://doi.org/10.1002/widm.8 
Loh, W.-Y. 2002. Regression trees with unbiased variable selection and interaction detection, Statistica Sinica 12(2): 361-386.

Long, K.; Wu, H.; Zhang, Z.; Murphy, M. 2014. Quantitative Relationship between Crash Risks and Pavement Skid Resistance. Report No FHWA/TX-13/0-6713-1. Texas Department of Transportation, Austin, TX. 71 p. Available from Internet: http://library.ctr.utexas.edu/ctr-publications/0-6713-1.pdf

McGovern, C.; Rusch, P.; Noyce, D. A. 2011. State Practices to Reduce Wet Weather Skidding Crashes. Publication FHWASA-11-21. Federal Highway Administration, US Department of Transportation. 43 p. Available from Internet: http://safety.fhwa.dot.gov/speedmgt/ref_mats/fhwasa1121/ fhwasa1121.pdf

Noyce, D. A.; Bahia, H. U.; Yambo, J.; Chapman, J.; Bill, A. 2007. Incorporating Road Safety into Pavement Management: Maximizing Surface Friction for Road Safety Improvements. Report No. MRUTC 04-04. Wisconsin Department of Transportation, Madison, WI. 241 p. Available from Internet: http://www.wistrans.org/mrutc/files/04-04_ MRUTC_FR.pdf

Ord, J. K.; Getis, A. 1995. Local spatial autocorrelation statistics: distributional issues and an application, Geographical Analysis 27(4): 286-306. http://doi.org/10.1111/j.1538-4632.1995.tb00912.x

Qin, X.; Khan, G.; Noyce, D. A. 2007. Spatial statistical approach to identifying snow crash-prone locations, in TRB 86th Annual Meeting Compendium of Papers CD-ROM, 21-25 January 2007, Washington, DC. 1-20.

Vadakpat, G. C. 1994. Analytical Study of Surface Friction of Wisconsin Trunk Highway System Pavements: MSc Thesis. University of Wisconsin-Madison, Madison, WI. 146 p. 